\title{
ON HOLOMORPHIC EXTENSION OF FUNCTIONS ON SINGULAR REAL HYPERSURFACES IN $\mathbb{C}^{n}$
}

\author{
TEJINDER S. NEELON
}

(Received 3 January 2000)

\begin{abstract}
The holomorphic extension of functions defined on a class of real hypersurfaces in $\mathbb{C}^{n}$ with singularities is investigated. When $n=2$, we prove the following: every $C^{1}$ function on $\Sigma$ that satisfies the tangential Cauchy-Riemann equation on boundary of $\left\{(z, w) \in \mathbb{C}^{2}:|z|^{k}<P(w)\right\}, P \in C^{1}, P \geq 0$ and $P \not \equiv 0$, extends holomorphically inside provided the zero set $P(w)=0$ has a limit point or $P(w)$ vanishes to infinite order. Furthermore, if $P$ is real analytic then the condition is also necessary.
\end{abstract}

2000 Mathematics Subject Classification. 32D15, 32V05.

1. Introduction. The problem of holomorphic extensions of CR functions on nonsingular real hypersurfaces of $\mathbb{C}^{n}$ is classical and well investigated. See, for example, [2]. An optimal result in $\mathbb{C}^{2}$ was obtained by Trepreau [6] (see Section 4).

In this paper, the phenomenon of holomorphic extension of functions on singular real hypersurfaces $\Sigma$ is investigated. In analogy with the nonsingular case, we need to have the notion of tangential Cauchy-Riemann equations on $\Sigma$. More precisely, we need the notion of a CR (Cauchy-Riemann) function. We call a function on $\Sigma$ a CR function if it is continuous on $\Sigma$ and satisfies tangential Cauchy-Riemann equations on the nonsingular part of $\Sigma$.

The hypersurfaces considered in this paper are the ones that bound regions which can be swept with analytic disks, with singularities being precisely the points where these disks degenerate into points. Following [3], the approach then is to integrate the given CR function $f$ on the boundaries of these disks with the Cauchy Kernel, and obtain a holomorphic function $\tilde{f}$ defined in the interiors of the disks. The question then is, are the boundary values of $\tilde{f}$ given by $f$ ? The main result roughly states that, if the degenerate disks are parameterized by a set which is a uniqueness set for holomorphic functions in the direction transversal to analytic disks, then the function $\tilde{f}$ is indeed the holomorphic extension of $f$. When $n=2$, this condition is also necessary in the real analytic case.

2. The main result. Let $\Omega$ be a region in $\mathbb{C}^{n}$ containing 0 . Let $\Sigma=\{z \in \Omega: \rho(z)=0\}$ be a connected ( $2 n-1)$-dimensional subset of $\Omega$, where $\rho$ is a real-valued $C^{2}$ function on $\Omega$ such that $\rho(0)=0$ and $d \rho \neq 0$ on $\Sigma$. For $z=\left(z_{1}, \ldots, z_{n}\right) \in \mathbb{C}^{n}$, let $z^{\prime}=\left(z_{2}, \ldots, z_{n}\right)$, and let $\pi^{\prime}$ denote the projection $\pi^{\prime}(z)=z^{\prime}$. For each fixed $z_{0}^{\prime} \in \pi^{\prime}(\Omega)$, put

$$
\gamma_{z_{0}^{\prime}}=\left\{z_{1}: \rho\left(z_{1}, z_{0}^{\prime}\right)=0\right\} .
$$

We assume that $\Sigma$ satisfies the following hypothesis: each $\gamma_{z_{0}^{\prime}}$ either degenerates into 
a point or it defines a closed connected $C^{1}$ curve in the $z_{1}$-plane such that

$$
\rho_{z_{1}}\left(z_{1}, z_{0}^{\prime}\right) \neq 0 \quad \forall z_{1} \in \gamma_{z_{0}^{\prime}} .
$$

Put

$$
\begin{gathered}
S_{\Sigma}=\{p \in \Sigma: d \rho(p)=0\}, \\
D_{\Sigma}=\left\{z^{\prime} \in \pi^{\prime}(\Omega): \gamma_{z^{\prime}} \text { degenerates }\right\} .
\end{gathered}
$$

LEMMA 2.1. If $\Sigma, D_{\Sigma}$, and $S_{\Sigma}$ are as above, then the following hold:

$$
\begin{gathered}
D_{\Sigma}=\pi^{\prime}\left\{z \in \Sigma: \rho_{\bar{z}_{1}}(z)=0\right\}, \\
D_{\Sigma} \neq \pi^{\prime}(\Omega) \\
\pi^{\prime}\left(S_{\Sigma}\right) \subset D_{\Sigma} .
\end{gathered}
$$

Proof. Suppose $z_{0}^{\prime} \in D_{\Sigma}$. If $\left\{z_{1}^{0}\right\}=\gamma_{z_{0}^{\prime}}$ then $\left(z_{1}^{0}, z_{0}^{\prime}\right) \in \Sigma$, and if $\rho_{\bar{z}_{1}}\left(z_{1}^{0}, z_{0}^{\prime}\right) \neq 0$ then the equation $\rho\left(z_{1}, z_{0}^{\prime}\right)=0$ defines a one-dimensional curve near $z_{1}=z_{1}^{0}$, which is a contradiction.

Conversely, assume $z^{0} \in \Sigma$ is such that $\rho_{\bar{z}_{1}}\left(z^{0}\right)=0$. Since $\rho$ is real, $\rho_{z_{1}}\left(z^{0}\right)=0$ also. If $z_{0}^{\prime}=\pi^{\prime}\left(z^{0}\right) \notin D_{\Sigma}$ then, by the hypothesis on $\Sigma, \rho\left(z_{1}, z_{0}^{\prime}\right)=0$ is the defining equation of a closed connected $C^{1}$ curve in $z_{1}$-space, which is a contradiction since $d \rho\left(\cdot, z_{0}^{\prime}\right) \equiv 0$.

To prove (2.5), assume that $D_{\Sigma}=\pi^{\prime}(\Omega)$. Let $\alpha\left(z^{\prime}\right)$ denote the unique solution of $\rho\left(\alpha, z^{\prime}\right)=0$. Note that $\alpha$ is at least continuous. Now since the map

$$
z^{\prime} \longrightarrow\left(\alpha\left(z^{\prime}\right), z^{\prime}\right)
$$

parameterizes $\Sigma$, the real-dimension of $\Sigma$ is $2 n-2$. This is a contradiction to our hypothesis that $\Sigma$ is $(2 n-1)$-dimensional.

Finally, (2.6) follows immediately from (2.4).

The vector fields

$$
L_{j}=\rho_{\bar{z}_{j}} \frac{\partial}{\partial \bar{z}_{1}}-\rho_{\bar{z}_{1}} \frac{\partial}{\partial \bar{z}_{j}}, \quad 2 \leq j \leq n,
$$

have continuous coefficients which vanish precisely at the singular set $S_{\Sigma}$, and form a basis of tangential Cauchy-Riemann equations on the nonsingular part of $\Sigma$.

Let $\Sigma^{ \pm}$denote the two "sides" of $\Sigma$. By replacing $\rho$ by $-\rho$, if necessary, we may assume that

$$
\Sigma^{+}=\{z \in \Omega: \rho(z)<0\}
$$

is the side that contains the interiors of the curves $\gamma_{z^{\prime}}$.

Definition 2.2. A $C^{1}$ function $f$ defined on $\Sigma$ is called a CR function if it satisfies,

$$
L_{j} f \equiv 0 \quad \text { on the regular part of } \Sigma \forall j=2, \ldots, n .
$$

(Here by a $C^{1}$ function on $\Sigma$ we mean a function continuous on $\Sigma$, and $C^{1}$ on $\Sigma-S_{\Sigma}$.)

LEMMA 2.3. If $u$ is a $C R$ function on $\Sigma$, then

$$
\tilde{u}\left(z^{\prime}\right)=\int_{\rho\left(\zeta, z^{\prime}\right)=0} u\left(\zeta, z^{\prime}\right) d \zeta
$$

is a holomorphic function in $\pi^{\prime}(\Omega)$. 
Proof. It is clear that $\tilde{u}$ is continuous and

$$
\tilde{u}\left(z^{\prime}\right)=0 \quad \text { on } D_{\Sigma} .
$$

By Rado's theorem [5] and by Hartogs's theorem, it is enough to prove that $\tilde{u}$ is separately holomorphic in $\pi^{\prime}(\Omega)-D_{\Sigma}$. It is enough to show that it is holomorphic in one of the variables, say $z_{2}$, with the remaining variables fixed. Denote $z^{\prime \prime}=\left(z_{3}, \ldots, z_{n}\right)$ and fix $z^{\prime \prime}=z_{0}^{\prime \prime}$. For the sake of convenience of notations, in the rest of the proof of Lemma 2.3, we omit the $z_{0}^{\prime \prime}$ and write $\rho\left(z_{1}, z_{2}\right)$ for $\rho\left(z_{1}, z_{2}, z_{0}^{\prime \prime}\right)$. Observe that the induced tangential Cauchy-Riemann vector field on $\rho\left(z_{1}, z_{2}\right)=0$ is

$$
L_{2}=\rho_{\bar{z}_{2}} \frac{\partial}{\partial \bar{z}_{1}}-\rho_{\bar{z}_{1}} \frac{\partial}{\partial \bar{z}_{2}}
$$

Let $C$ be an arbitrary closed rectifiable Jordan curve in the $z_{2}$-space such that $\left(z_{2}, z_{0}^{\prime \prime}\right) \notin$ $D_{\Sigma}$ for all $z_{2} \in \overline{\operatorname{int}(C)}$, the closure of the interior. If $z_{1}=\gamma\left(\theta, z_{2}\right)$ denotes the $C^{1}$ curve $\rho\left(z_{1}, z_{2}^{0}\right)=0$, then

$$
\rho\left(\gamma, z_{2}\right) \equiv 0 .
$$

By Morera's theorem, to prove the holomorphicity of $\tilde{u}$ it is enough to show that

$$
\int_{C} \tilde{u} d z_{2}=0
$$

If we view $d z_{1}$ and $d \bar{z}_{1}$ as $d y$ and $d \bar{\gamma}$, restricted to $\Sigma$, respectively, then on $\Sigma$ we have

$$
\rho_{z_{1}} d z_{1}+\rho_{\bar{z}_{1}} d \bar{z}_{1}+\rho_{z_{2}} d z_{2}+\rho_{\bar{z}_{2}} d \bar{z}_{2}=0 .
$$

Since $\rho_{\bar{z}_{1}} \neq 0$, we have

$$
d \bar{z}_{1} \wedge d z_{1} \wedge d z_{2}=-\frac{\rho_{\bar{z}_{2}}}{\rho_{\bar{z}_{1}}} d \bar{z}_{2} \wedge d z_{1} \wedge d z_{2}
$$

Since $L_{2} u=0$, the above equation implies that

$$
d\left(u(z) d z_{1} \wedge d z_{2}\right)=\left(\frac{\partial u}{\partial \bar{z}_{1}} d \bar{z}_{1}+\frac{\partial u}{\partial \bar{z}_{2}} d \bar{z}_{2}\right) d z_{1} d z_{2}=\frac{1}{\rho_{\bar{z}_{1}}}\left(L_{2} u\right) d \bar{z}_{2} d z_{1} d z_{2}=0 .
$$

Hence the restriction of the form $u(z) d z_{1} \wedge d z_{2}$ to $\Sigma$ is closed. By applying the Stokes' theorem, we have

$$
\int_{C} \tilde{u} d z_{2}=0
$$

DEFINITION 2.4. $\Sigma$ is said to be extendible to the side $\Sigma^{+}$if the following holds: for every CR function $f$ on $\Sigma \cap \Omega$, there is a function $F$ holomorphic in $\Sigma^{+}$and continuous on $\overline{\Sigma^{+}}$such that $\left.F\right|_{\Sigma \cap \Omega}=f$.

By a uniqueness set $E$ of a connected and simply connected set $U$, we mean a subset $E \subseteq \bar{U}$ such that for any function $f$ continuous on $\bar{U}$ and holomorphic in $U$ that vanishes on $E$ vanishes identically on $U$.

THEOREM 2.5. Let $\Sigma$ and $D_{\Sigma}$ be as described above. If $D_{\Sigma}$ is a uniqueness set of $\pi^{\prime}(\Omega)$, then $\Sigma$ is extendible to $\Sigma^{+}$. 
Proof. Let $f$ be a CR function on $\Sigma$. Following [3], we define

$$
\tilde{f}(z)=(2 \pi i)^{-1} \int_{\rho\left(\zeta_{1}, z^{\prime}\right)=0} \frac{f\left(\zeta_{1}, z^{\prime}\right)}{\zeta_{1}-z_{1}} d \zeta_{1} .
$$

Since for $z \notin \Sigma$, the integrand in $\tilde{f}$ is a CR function on $\rho\left(\zeta_{1}, z^{\prime}\right)=0$ we can apply Lemma 2.3 to conclude that $\tilde{f}$ is holomorphic in the domain $\Omega-\Sigma$.

It needs to be shown that $\tilde{f}$ is an extension of $f$ to $\Sigma^{+}$. It is enough to show this for each fixed $z^{\prime}=z_{0}^{\prime}$. In order to show that $\tilde{f}$ is a holomorphic extension of $f$ to the inside of $\rho\left(z_{1}, z_{0}^{\prime}\right)=0$, all we need to show is that $\tilde{f}\left(z_{1}, z^{\prime}\right)=0$, for all $\left(z_{1}, z^{\prime}\right)$ such that $z_{1} \notin \overline{\operatorname{int}\left(\gamma_{z^{\prime}}\right)}$. (See [4].)

Let $\pi_{1}$ denote the projection of $\mathbb{C}^{n}$ to the first coordinate: $\pi_{1}(z)=z_{1}$. Let $\left\{K_{j}\right\}_{j=1}^{\infty}$ be a sequence of exhausting sequence of compact open subsets of $\pi_{1}(\Omega)$. For each $j \geq 1$, put

$$
\Omega_{j}=K_{j} \times \pi^{\prime}(\Omega) .
$$

We first show that the restriction of $f$ to $\Sigma \cap \Omega_{j}$ extends holomorphically to $\Sigma_{j}^{+}=$ $\Omega_{j} \cap \Sigma^{+}$. Let $R>0$ be a large constant such that the following is satisfied:

$$
\left|z_{1}\right|>R \Longrightarrow\left(z_{1}, z^{\prime}\right) \notin \Omega_{j} \quad \forall z^{\prime} \in \pi^{\prime}(\Omega) .
$$

Let $z_{1}^{0},\left|z_{1}^{0}\right|>R$, be fixed. By (2.22) and by Lemma 2.3, it follows that $z^{\prime} \rightarrow \tilde{f}\left(z_{1}^{0}, z^{\prime}\right)$ is holomorphic in $\pi^{\prime}(\Omega)$, and it clearly vanishes on $D_{\Sigma}$. By the hypothesis that $D_{\Sigma}$ is a uniqueness set of $\pi^{\prime}(\Omega), \tilde{f}\left(z_{1}^{0}, z^{\prime}\right)$ must vanish everywhere in $\pi^{\prime}(\Omega)$. Hence

$$
\tilde{f}\left(z_{1}, z^{\prime}\right) \equiv 0 \quad \text { if }\left|z_{1}\right|>R .
$$

Since $\tilde{f}$ is holomorphic in $\rho(z)>0, \tilde{f}\left(z_{1}, z^{\prime}\right)=0$ for all $z_{1} \in K_{j}$ and outside $\gamma_{z^{\prime}}$.

If $\tilde{f}_{j}$ denotes the holomorphic extension of $f$ to $\Omega_{+}^{(j)}$. It is clear from the construction of $\tilde{f}_{j}$ 's that $\tilde{f}_{j}=\tilde{f}_{j^{\prime}}$ if $j^{\prime}>j$. By letting $j \rightarrow \infty$, we get the extension to all of $\Omega$.

\section{The two-dimensional case}

THEOREM 3.1. Let $\Omega \subset \mathbb{C}^{2}$ be a region containing the origin. Let $P \in C^{1}\left(\pi_{w}(\Omega)\right)$ such that $P \geq 0$ and $P \neq 0$. Let $k>1$. Consider the following three-dimensional set:

$$
\Sigma=\left\{(z, w) \in \Omega:|z|^{k}=P(w)\right\},
$$

then, in order for $\Sigma$ to be extendible to

$$
\left\{(z, w) \in \Omega:|z|^{k}<P(w)\right\},
$$

it is sufficient that either the zero ser $P(w)=0$ has a limit point in $\pi_{w}(\Omega)$ or $P(w)$ is flat at some point in $\pi_{w}(\Omega)$. Furthermore, if $P$ is real analytic then the above condition is also necessary.

Proof. The vector field

$$
L=2 P_{\bar{w}} \frac{\partial}{\partial \bar{z}}-k z|z|^{k-1} \frac{\partial}{\partial \bar{w}}
$$

can be taken as a CR vector field on $\Sigma$. The intersection of each complex line $w=w_{0}$ with $\Sigma$ is a circle, possibly degenerate, centered at 0 in the $z$-plane. The degenerate 
ones are given by those $w \in D_{\Sigma}=\left\{w \in \pi_{w}(\Omega): P(w)=0\right\}$. Hence, if $P(w)=0$ has a limit point, then $\Sigma$ satisfies the hypothesis of Theorem 2.5 .

Now assume that $D_{\Sigma}$ is discrete and $P$ vanishes to infinite order at $w_{0} \in D_{\Sigma}$. For the sake of convenience we assume that $w_{0}=0$.

As in the proof of Theorem 2.5, the function

$$
\tilde{f}(z, w)=(2 \pi i)^{-1} \int_{|z|=p(w)} \frac{f(\zeta, w)}{\zeta-z} d \zeta
$$

is an holomorphic extension of $f$ to $|z|<p(w)$, provided

$$
J_{m}(w)=\int_{|z|=p(w)} f(\zeta, w) \zeta^{m} d \zeta \equiv 0 \quad \forall m=0,1,2, \ldots
$$

By following the reasoning in the proof of Lemma 2.3, and by using Rado's theorem, we can conclude that the $J_{m}$ 's are holomorphic functions in $\pi_{w}(\Omega)$. We show that the $J_{m}$ 's vanish to infinite order at 0 .

Let $m$ be fixed. Rewriting $J_{m}$ as

$$
J_{m}(w)=i(p(w))^{m+1} \int_{0}^{2 \pi} f\left(p(w) e^{i \theta}, w\right) e^{i(m+1) \theta} d \theta
$$

we have

$$
\left|J_{m}(w)\right| \leq|p(w)|^{m+1} \int_{0}^{2 \pi}\left|f\left(p(w) e^{i \theta}, w\right)\right| d \theta .
$$

The integrand in $J_{m}$ is bounded when $w$ stays in a bounded set containing 0 . Since $P$ vanishes to infinite order at 0 , for every integer $l>0$ there is a constant $C_{l}$ such that $|P(w)|<C_{l} \cdot|w|^{l}$. We have

$$
|J(w)|<C_{l}^{1 / k} \cdot|w|^{l(m+1) / k}(2 \pi) \sup _{|w|<R, \theta}\left|f\left(p(w) e^{i \theta}, w\right)\right|<C_{l}^{\prime}|w|^{l^{\prime}},
$$

where $C_{l^{\prime}}^{\prime}>0$ is a constant, and $l^{\prime}$ is an integer, $l^{\prime} \rightarrow \infty$ as $l \rightarrow \infty$. Hence, $J_{m}$ vanishes to infinite order at 0 .

Suppose $P$ is real analytic. First assume that $P(w) \neq 0$ for $w \neq 0$. Since $P$ is real analytic, there are constants $C>0$ and $\delta>0$ such that $|P(w)| \geq C|w|^{\delta}$.

Let $m$ be a large fixed positive integer, and let $u$ be a function on $\Sigma$ defined as follows:

$$
u(z, w)=\left.\frac{w^{m}}{z^{k}}\right|_{\Sigma} \text { if } 0 \neq(z, w) \in \Sigma \text {, if } u(0,0)=0 .
$$

Since

$$
|u|=\left.\frac{|w|^{m}}{|z|^{k}}\right|_{\Sigma}=\frac{|w|^{m}}{P(w)} \leq|w|^{m-\delta}
$$

it follows that it is continuous on $\Sigma$. By choosing $m$ large, we can make $u$ a $C^{r}$ function for a given $r$. Clearly $u$ is a CR function that does not extend holomorphically to the side $|z|^{k}<P(w)$. This example can be easily modified to the case when $P(w)$ has more than one isolated zero.

4. Examples. A theorem of Trepreau [6] says that if a nonsingular real hypersurface $\Sigma \subset \mathbb{C}^{2}, 0 \in \Sigma$, does not contain a holomorphic curve passing through 0 , then $\Sigma$ is 
extendible, near 0 , to at least one side. The analogue of this theorem does not hold for singular real hypersurfaces as shown by the following example.

EXAMPLE 4.1. Consider the hypersurface

$$
\Sigma=\left\{(z, w) \in \mathbb{C}^{2} ;|z|^{2}=|w|^{2}+|w|^{4}\right\}
$$

with an isolated singularity at the origin. It follows from Theorem 3.1, that $\Sigma$ is not extendible to either side. Suppose $V$ is a holomorphic curve contained in $\Sigma$. If $p \neq(0,0)$ is a nonsingular point of $V$, then $\Sigma$ is of infinite type at $p$ (see [1]). But a simple calculation shows that $\Sigma$ is of type 2 at all points $p \neq(0,0)$. Hence, $\Sigma$ does not contain a holomorphic curve.

EXAMPLE 4.2. Theorem 3.1 shows that the hypersurface

$$
\Sigma=\left\{(z, w) \in \mathbb{C}^{2}:|z|^{2}=w^{2}+\bar{w}^{2}\right\}
$$

is extendible to the side $|z|^{2}<w^{2}+\bar{w}^{2}$. As before it is easy to see that the restriction of $z^{4} w^{-1}$ to $\Sigma$ is a CR function which clearly does not extend holomorphically to $|z|^{2}>w^{2}+\bar{w}^{2}$.

EXAMPLE 4.3. The hypersurface $\Sigma=\left\{(z, w) \in \mathbb{C}^{2} ;|z|^{2}=e^{-1 /|w|^{2}}\right\} \cup\{(0,0)\}$ has an isolated singularity at the origin and, by Theorem 3.1, is extendible to $|z|^{2}<e^{-1 /|w|^{2}}$.

\section{REFERENCES}

[1] T. Bloom and I. Graham, On "type" conditions for generic real submanifolds of $\mathrm{C}^{n}$, Invent. Math. 40 (1977), no. 3, 217-243. MR 58\#28644. Zbl 346.32013.

[2] A. Boggess, CR Manifolds and the Tangential Cauchy-Riemann Complex, Studies in Advanced Mathematics, CRC Press, Florida, 1991. MR 94e:32035. Zbl 760.32001.

[3] H. Lewy, On the local character of the solutions of an atypical linear differential equation in three variables and a related theorem for regular functions of two complex variables, Ann. of Math. (2) 64 (1956), 514-522. MR 18,473b. Zbl 074.06204.

[4] N. I. Muskhelishvili, Singular Integral Equations. Boundary Problems of Function Theory and Their Application to Mathematical Physics, P. Noordhoff N. V., Groningen, Holland, 1953. MR 15,434e. Zbl 051.33203.

[5] W. Rudin, Function Theory in the Unit Ball of $\mathbf{C}^{n}$, Grundlehren der Mathematischen Wissenschaften [Fundamental Principles of Mathematical Science], vol. 241, SpringerVerlag, New York, 1980. MR 82i:32002. Zbl 495.32001.

[6] J. M. Trépreau, Sur le prolongement holomorphe des fonctions C-R défines sur une hypersurface réelle de classe $C^{2}$ dans $\mathbf{C}^{n}$ [The holomorphic extension of $C R$ functions defined on a real $C^{2}$ hypersurface in $\mathbf{C}^{n}$ ], Invent. Math. 83 (1986), no. 3, 583-592 (French). MR 87f:32035. Zbl 586.32016.

Tejinder S. NeElon: DePartment of MATHEMATics, CAlifornia State University SAN MARCOS, SAN MARCOS, CA 92096, USA

E-mail address: nee1on@csusm.edu 


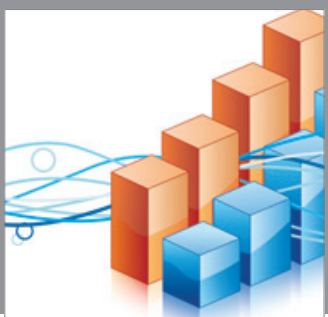

Advances in

Operations Research

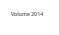

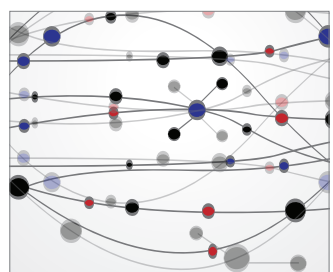

\section{The Scientific} World Journal
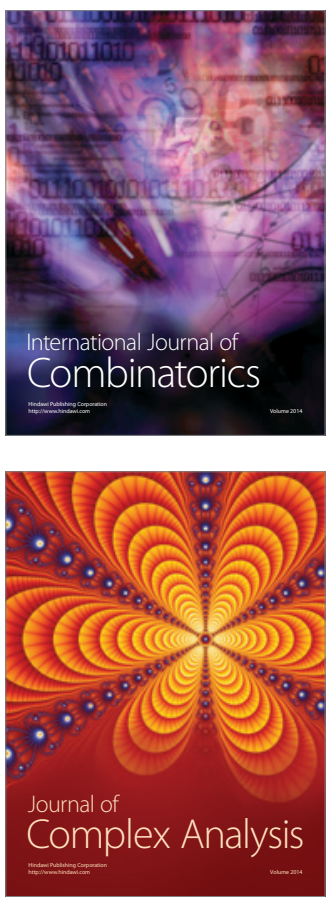

International Journal of

Mathematics and

Mathematical

Sciences
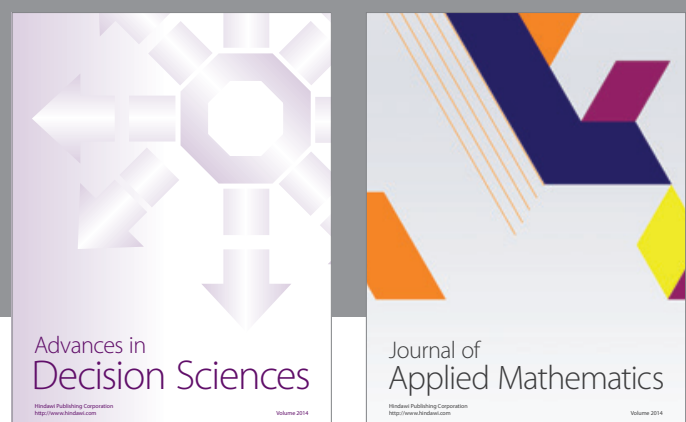

Journal of

Applied Mathematics
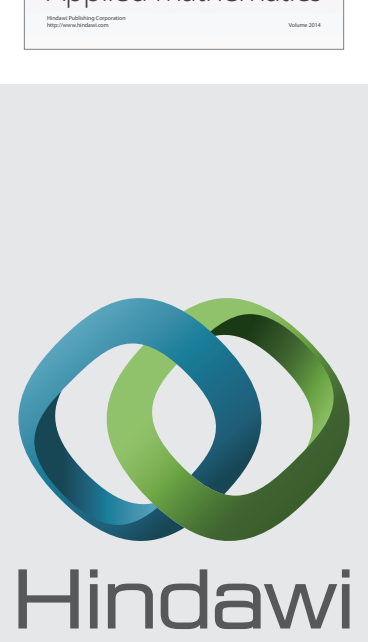

Submit your manuscripts at http://www.hindawi.com
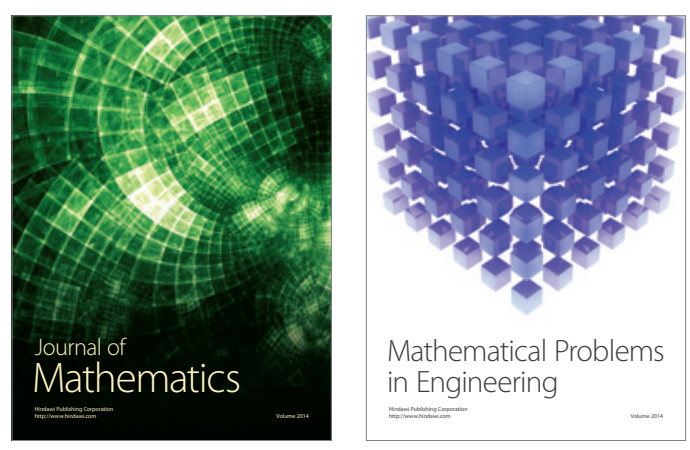

Mathematical Problems in Engineering
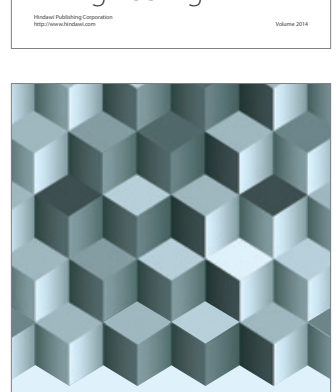

Journal of

Function Spaces
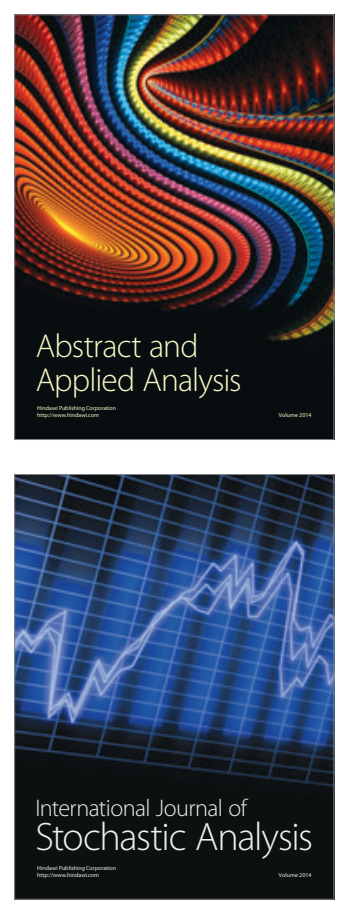

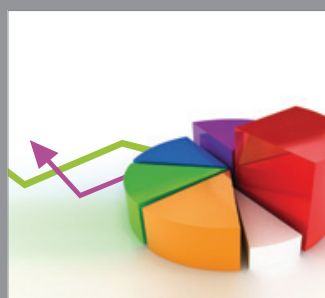

ournal of

Probability and Statistics

Promensencen
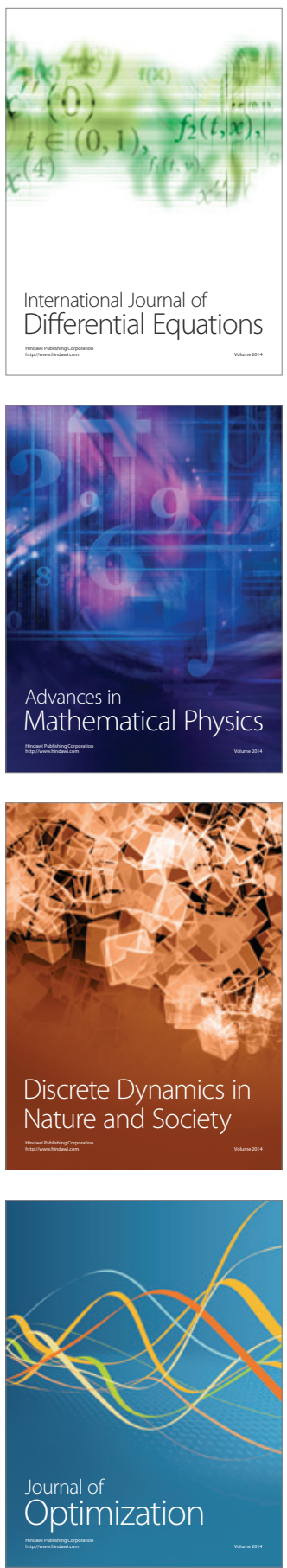